\title{
Desafios para a práxis do docente em Enfermagem no Ensino Superior
}

\author{
Challenges for teachers' practice in Nursing in Higher Education \\ Desafíos para la práctica de los docentes en la Enfermería en la Enseñanza Superior
}

Recebido: 01/02/2021 | Revisado: 10/02/2021 | Aceito: 31/03/2021 | Publicado: 09/04/2021

\author{
Samara Letícia Mendonça Pereira \\ ORCID: https://orcid.org/0000-0003-2835-5316 \\ Universidade de São Paulo, Brasil \\ E-mail: samara.leticia@usp.br \\ Daiane de Souza Fernandes \\ ORCID: https://orcid.org/0000-0001-6629-4222 \\ Universidade de São Paulo, Brasil \\ E-mail: daissf@usp.br \\ Thalita Lins Soares Silveira \\ ORCID: https://orcid.org/0000-0002-0518-1619 \\ Universidade de São Paulo, Brasil \\ E-mail: thalita.lins@usp.br \\ Adriana Inocenti Miasso \\ ORCID: https://orcid.org/0000-0003-1726-7169 \\ Universidade de São Paulo, Brasil \\ E-mail: amiasso@eerp.usp.br \\ Rosalina Aparecida Partezani Rodrigues \\ ORCID: https://orcid.org/0000-0001-8916-1078 \\ Universidade de São Paulo, Brasil \\ E-mail: rosalina@eerp.usp.br \\ Sandra Cristina Pillon \\ ORCID: https://orcid.org/0000-0001-8902-7549 \\ Universidade de São Paulo, Brasil \\ E-mail: pillon@eerp.usp.br
}

\begin{abstract}
Resumo
Objetivo: refletir sobre os desafios para a práxis do docente em enfermagem no ensino superior. Metodologia: trata-se de estudo reflexivo, fundamentado na literatura científica brasileira e internacional. Partiu-se de três seções temáticas: i) A construção da identidade profissional do docente no ensino superior; ii) A formação pedagógica do docente em enfermagem no ensino superior; iii) A dicotomia entre ensino e assistência. Resultados: o ingresso na docência de educação superior é circundado por diversos desafios, como a construção da identidade profissional, processo de formação pedagógica e a dicotomia entre ensino e assistência. A formação na área não garante ferramentas suficientes para atuação como professor, caracterizando temáticas que urgem discussões sobre as exigências para este campo de atuação profissional. Considerações Finais: evidencia-se a indispensabilidade de processos formativos que garantam preparo frente a diversas complexidades que envolvem o educar e o formar profissionais para a docência superior. Portanto, esta reflexão contribui acrescentando informações pertinentes que poderão auxiliar em formações científicas e educação permanente para a reorganização de conceitos e ideias atrelados aos desafios para a práxis do docente em enfermagem no ensino superior.
\end{abstract}

Palavras-chave: Ensino; Enfermagem; Docente de enfermagem; Ensino superior; Educação permanente.

\begin{abstract}
Objective: to reflect on the challenges for the teaching practice of nursing in higher education. Methodology: this is a reflective study, based on Brazilian and international scientific literature. It is based on three thematic sections: i) The construction of the professional identity of the teacher in higher education; ii) The pedagogical formation of the teacher in nursing in higher education; iii) The dichotomy between teaching and care. Results: entry into higher education teaching is surrounded by various challenges, such as the construction of professional identity, the pedagogical training process and the dichotomy between teaching and care. Training in this area does not guarantee sufficient tools to act as a teacher, characterizing themes that urgently require discussions on the requirements for this professional field. Final Considerations: the indispensability of training processes that guarantee preparation for the various complexities involved in educating and training professionals for higher education is evident. Therefore, this reflection contributes adding pertinent information which may help in scientific formations and permanent education for the reorganization of concepts and ideas linked to the challenges for the practice of the teacher in nursing in higher education.
\end{abstract}

Keywords: Teaching; Nursing; Nursing teacher; Higher Education; Continuing education. 


\begin{abstract}
Resumen
Objetivo: reflexionar sobre los retos de la práctica docente de la enfermería en la enseñanza superior. Metodología: se trata de un estudio reflexivo, basado en la literatura científica brasileña e internacional. Se han iniciado tres apartados temáticos: i) La construcción de la identidad profesional del profesor en la enseñanza superior; ii) La formación pedagógica del profesor de enfermería en la enseñanza superior; iii) La dicotomía entre la enseñanza y el cuidado. Resultados: el ingreso a la docencia en la educación superior está rodeado de varios desafíos, como la construcción de la identidad profesional, el proceso de formación pedagógica y la dicotomía entre docencia y atención. La formación en el área no garantiza las herramientas suficientes para actuar como docente, caracterizando temas que requieren urgentemente discusiones sobre los requisitos para este campo de acción profesional. Consideraciones finales: es evidente la indispensabilidad de procesos de formación que garanticen la preparación para las diversas complejidades que implica la educación y formación de profesionales para la educación superior. Por lo tanto, esta reflexión contribuye a añadir información pertinente que puede ayudar en la formación científica y la educación continua para la reorganización de los conceptos e ideas vinculadas a los desafíos para la práctica de la enseñanza de la enfermería en la educación superior.
\end{abstract}

Palabras clave: Enseñanza; Enfermería; Profesor de enfermería; Educación superior; Educación continua.

\title{
1. Introdução
}

A fragilidade de atenção a formação inicial pedagógica do professor enfermeiro universitário para exercício de uma docência em sintonia com os desafios contemporâneos da atualidade, interfere diretamente nas interfaces do ensinar e do aprender, o que leva a refletir e a explorar mais este espaço, a partir de discussões e leituras realizadas na disciplina "A pósgraduação e o pós-graduando", do Programa de Pós-Graduação da Escola de Enfermagem de Ribeirão Preto da Universidade de São Paulo - USP.

As discussões e reflexões acerca da formação do professor que atua no ensino superior é constante na realidade atual. Nessa direção, são frequentes, porém pouco compreendidas, questões como: de que maneira o professor é preparado para exercer a docência? Este docente se sente preparado para ingressar no mundo acadêmico?

São prementes e necessárias reflexões sobre a práxis do docente em enfermagem no ensino superior, uma vez que diversos desafios como a construção da identidade profissional, processo de formação pedagógica e a dicotomia entre ensino e assistência, caracterizam temáticas que suscitam discussões para a atuação deste profissional.

Instituições de ensino superior constituem ambientes de construção, propagação e formação de saberes e culturas, portanto contribui na transformação social, em uma sociedade mais globalizada e em incessantes avanços (Lauxen, 2014).

Atualmente o ensino superior no Brasil, especialmente os cursos superiores de enfermagem, tem avançado e demonstrado crescimento acelerado, o que leva a refletir nas exigências para formação dos professores, dos futuros profissionais de enfermagem e também dos egressos. Como consequência dessa expansão, constata-se o aumento de ofertas na docência, com ingresso de enfermeiros advindos de bacharel em enfermagem e com apenas titulação mínima lato sensu (Agnelli \& Nakayama, 2018).

Nas diretrizes curriculares dos cursos de graduação em enfermagem, o educar no cenário da saúde faz parte do processo de trabalho do enfermeiro, todavia acredita-se que esse processo se torna pouco explorado pelos profissionais, devido à alta demanda dentro da prática curativa, priorizando assim a assistência hospitalar e não o ensino. Diante disso, percebe-se a ausência durante a graduação de disciplinas pedagógicas para a atuação na docência no ensino em enfermagem, visto que os docentes passam a utilizar os conhecimentos da prática e as referências acadêmicas passadas, o que corrobora para formação de profissionais de uma área técnica, migrando para docência com experiência apenas na assistência (Brasil, 2001; Braga \& Boas, 2014).

Os anos iniciais de docência podem ser caracterizados por um período de novidades, superação e exploração relacionadas, principalmente, ao impacto inicial e a complexidade profissional, uma vez que existe a mudança do posto de aluno para professor. Todavia, o choque de realidade relacionado às diferenças entre a formação e a atuação profissional, pode resultar em medos e inseguranças individuais e coletivas no contexto do ensino. Desse modo, podem coexistir nessa fase 
inicial o entusiasmo de início de carreira e por fazer parte de um corpo profissional, a ansiedade pelas responsabilidades que lhe são designadas (Leenknecht, Wijnia, Loyens \& Rikers, 2017; Rabelo \& Monteiro, 2019)

Novas diligências relacionadas ao processo de ensino e aprendizagem em enfermagem apontam para necessidade desta área da saúde realizar interlocuções com outras áreas de conhecimento com um olhar interdisciplinar, e isto requer pensar na identidade profissional do docente Enfermeiro (Braga \& Boas, 2014).

Existe também a necessidade de desenvolver ambientes mais adequados para o processo de aprendizagem do professor, tanto para a formação inicial, quanto para aqueles que já ingressaram, uma vez que no Brasil, o ensino ainda é dissociado, ou seja, a tríade ensino médio, superior e pesquisa não se comunicam, e essa segregação tem como consequência uma formação defasada e com professores despreparados (Nóvoa, 2019).

Com isso, ressalta-se que o ensino está diretamente voltado para a formação científica, pedagógica e política, bem como para a indissociabilidade entre ensino, pesquisa e extensão (Emmel \& Krul, 2017).

Estudos sobre a atuação do enfermeiro docente são importantes, visto que a formação na área não garante ferramentas suficientes para atuação como professor, sugerindo a necessidade de debates sobre esta temática.

Portanto, este artigo objetiva refletir sobre os desafios para a práxis do docente em enfermagem no ensino superior.

\section{Metodologia}

Trata-se de um estudo de cunho reflexivo, oriundo de debates na disciplina "A pós-graduação e o pós-graduando: formação e pesquisa", do Programa de Pós-Graduação a nível de mestrado e doutorado da Escola de Enfermagem de Ribeirão Preto da Universidade de São Paulo (USP). A disciplina foi ministrada no período de fevereiro de 2020, propiciando amplas discussões sobre o processo de formação do pós-graduando para a pesquisa e docência, aguçando uma reflexão mais aprofundada sobre os desafios para a práxis do enfermeiro docente, fundamentada em leituras minuciosas e críticas da literatura científica brasileira e internacional e, além da vivência de algumas autoras com a temática em destaque.

Como não se trata de um estudo de revisão, os estudos selecionados para leitura densa foram eleitos a partir da indicação de leituras durante o desenvolvimento da disciplina e seleção por algumas autoras devido a experiência com o tema.

Os artigos de reflexão se aproximam de estudos de abordagem qualitativa, no sentido de descrição e análise de elementos teóricos alcançados através do levantamento bibliográfico (Minayo, 2006).

Foi estruturado por meio de três seções temáticas, possibilitando apresentar pertinentes discussões baseadas em uma breve caracterização dos desafios enfrentados para o exercício da docência em enfermagem e um debate para a atuação do enfermeiro no ensino superior. As seç̃es são: i) A construção da identidade profissional do docente no ensino superior; ii) A formação pedagógica do docente em enfermagem no ensino superior; iii) A dicotomia entre ensino e assistência.

\section{Resultados e Discussão}

\subsection{A construção da identidade profisssional do docente no ensino superior}

A identidade profissional do docente no ensino superior a muito tempo é caracterizada pelas dimensões principais de habilidades relacionadas ao ensino e pesquisa. Salienta-se que as universidades nas últimas décadas, vem passando por diversas mudanças históricas como, demandas igualitárias, necessidade de treinamento, transformações das funções institucionais e mudanças políticas e econômicas, fatores estes que influenciam na identidade do professor (Coggi \& Ricchiardi, 2018).

Estas questões demandaram uma necessidade de habilidades para a construção da identidade profissional do docente, relacionadas às atividades organizacionais, gerenciais e sociais (Coggi \& Ricchiardi, 2018; Wenlu \& Wang, 2015). 
À medida que a qualidade do ensino superior se torna o foco de atenção no mundo ocidental e a demanda por ensino e aprendizagem aumenta, elucida-se também a exigência de investimento na formação de professores universitários (Silander \& Stigmar, 2019).

$\mathrm{O}$ ingresso na carreira docente no ensino superior muitas vezes é permeado por diversos desafios. Outro fator que influencia na construção da identidade profissional do professor é a busca por mudanças relacionadas a sua formação educacional direcionada a uma perspectiva mais dialógica e integrada, ressaltando-se que as principais fontes desta construção ainda são a experiência e inspiração em outros professores (Braga \& Boas, 2014; Fernandes \& Souza, 2017).

Portanto, esta identidade é entendida como um processo marcado pela trajetória de vida deste professor ou pelo grupo no qual estava inserido. Compreende uma extensa rede afetiva de relações que permearam a vida deste professor (Emmel \& Krul, 2017).

Outras causas que possuem relações inerentes com a construção da identidade profissional e ingresso na carreira do magistério superior, são justificativas relacionadas ao acaso, o qual permeia o início desta atividade profissional, sendo que um contingente pequeno de professores expressa um desejo ou plano estratégico para adentrar na profissão, o que pode estar relacionado ao processo de formação deste profissional, voltado para atuação específica em sua área (Braga \& Boas, 2014; Fernandes \& Souza, 2017).

A entrada efetiva no magistério superior nem sempre é uma escolha pessoal, pode estar atrelada a percursos momentâneos, circunstâncias que levaram este professor a atuar nesta profissão. Com isto, destaca-se a importância da instituição de ensino superior no processo de continuidade da formação (Emmel \& Krul, 2017).

O docente apresenta como gênese em sua profissão as dimensões relacionais e humana, e isto requer um processo formativo que ultrapasse as barreiras de domínio teórico e prático, pois ele precisa trabalhar com o conhecimento de modo que possa refletir sobre a sua prática e fomentar atitudes reflexivas grupais (Moreira \& Ferreira, 2014).

Ressalta-se que as instituições possuem papel primordial neste processo formativo, pois entende-se que é permanente, devendo ser desenvolvido durante toda a trajetória deste professor (Emmel \& Krul, 2017), ou seja, a docência é um processo construído ao longo da carreira.

\subsection{A formação pedagógica do docente em enfermagem no ensino superior}

A docência requer um processo de formação pedagógica, antes ou durante a atuação deste professor e isto é premente para o desenvolvimento de um processo ensino-aprendizagem eficaz. Adentrar nesta carreira com pouca ou nenhuma formação constitui-se um grande desafio. A heterogeneidade no processo de ensinar requer habilidades deste docente o que pode influenciar no seu desenvolvimento profissional, pessoal e até mesmo institucional (Fernandes \& Souza, 2017). A eficácia do ensino e a qualidade dos professores são fatores importantes que afetam o desenvolvimento dos alunos (Chan, 2018).

O exercício da docência em enfermagem tem total relação com questões de formação que percorre por perspectivas sociais pautadas na habilitação e atuação do enfermeiro docente, seja esta inicialmente em cursos de graduação ou em formação direcionada para o ensino técnico ou superior (Agnelli \& Nakayama, 2018).

No estudo de Agnelli e Nakayama, (2018), compreendem que a formação pedagógica do enfermeiro deve ser obtida em cursos de graduação em licenciatura e em cursos de pós-graduação stricto sensu, como o mestrado e doutorado. Entretanto, outras pesquisas evidenciaram que professores enfermeiros considerados bem sucedidos adentram na carreira docente por meio das experiências assistenciais, buscando posteriormente formações pedagógicas (Araújo, Gebran \& Barros, 2016).

A formação docente em enfermagem além de estar relacionada com o processo de educação permanente em estratégias de ensino, deve estar imbricada com a função política e social do trabalho em saúde. 
O contínuo aperfeiçoamento do docente torna-se primordial para a efetivação do desenvolvimento de um magistério em enfermagem eficaz, condizente com as necessidades do discente para atuação como futuro profissional. A função docente exige um corpo de conhecimentos técnicos relacionados à efetivação do processo ensino-aprendizagem que configure atitudes libertadoras, reflexivas, com o desenvolvimento de competências técnicas e sociais que não se resumem a processos de reprodução conteudista (Braga \& Boas, 2014), deste modo, a formação pedagógica deste professor é fundamental para a concretização deste processo.

Os profissionais de enfermagem que atuam na docência compreendem a necessidade da formação continuada como dimensão de contribuição permanente nas atualizações das áreas de saúde e educação (Agnelli \& Nakayama, 2018). A prática pedagógica é considerada por eles como algo construído anteriormente, que é planejado, implementado e posto em prática cotidianamente no ambiente universitário (Ribeiro et al., 2018).

É relevante salientar que grande parte dos docentes iniciam as suas práticas de ensino com base em modelo tradicional biomédico-tecnicista, buscam a formação pedagógica somente após terem iniciado na carreira e, na maioria das vezes, é por meio de cursos que acessam capacitação voltada para a docência (Orosco \& Scheide, 2008).

Braga e Boas (2014), acreditam que a formação pedagógica do enfermeiro professor é fundamental na superação do modelo tradicional de ensino, pois é na formação que se identifica o cerne de superação. Assim, para o progresso e efetivação do ensino reflexivo, é relevante que os professores apresentem domínio das suas práticas, demonstrem capacidade resolutivas de problemas e de desenvolvimento intelectual, sendo impreterível compreender e discutir a formação continuada do exercício da docência de enfermagem.

A formação do docente é imprescindível, sendo através dela garantidas atualizações concretas, objetivas e inovadoras para que o professor consiga disseminar seus conhecimentos da melhor forma aos alunos, resultando no abandono do modelo de ensino tradicional e ampliando as possibilidades do aprender enquanto ensina. É crucial no momento da formação a reflexão crítica voltada ao uso das práticas pedagógicas (Ribeiro et al., 2018).

Acredita-se na escassez de formação pedagógica por parte de cursos da graduação e pós-graduação stricto sensu direcionada para a prática da docência de enfermagem, visto que os docentes para alcançarem resultados necessitam do conhecer das práticas e referências universitárias anteriores. O enfermeiro docente deve transpor o exercício da docência com compromisso político, educativo, social e ético, o que indica premências de formação pedagógica e educação permanente que consiga contextualizar o cenário político (Agnelli \& Nakayama, 2018).

Por outro lado, a formação atualmente não é tarefa fácil, é desafiador para as instituições de ensino de enfermagem, sobretudo a qualidade da formação não pode estar pautada somente em âmbito do aprender a aprender, do aprender a fazer, aprender a ser e conviver, mas é necessário está relacionada com a dimensão política e social em saúde (Fernandes \& Rebouças, 2013).

Neste contexto, a formação pedagógica para os docentes possibilita transformações no processo de ensinoaprendizagem, permitindo o progresso dos alunos, tornando-os sujeitos ativos e independentes no processo, bem como o avanço da qualidade dos egressos (Alexandre, Werneck, Chainça \& Cesarino, 2018).

\subsection{A dicotomia entre ensino e assistência}

Um outro desafio que permeia atuação do docente de enfermagem na educação superior é uma bifurcação entre ensino e serviço, separação estabelecida que promove um distanciamento entre teoria e prática, promovendo um processo de fragilização na profissão, pois estabelece uma condição de sobreposição do ensino em relação a assistência, salientando-se que são atividades complementares e inerentes (Braga \& Boas, 2014). 
Ser enfermeiro não é o mesmo que ser professor de enfermagem, os conhecimentos empregados para cada uma dessas funções diferem-se na estrutura, o ensino para o ser professor relaciona-se a um processo identitário e específico. Além do mais, quando se pensa nesta questão a tendência é os professores transmitirem o conhecimento da forma como foram ensinados, perpetuando práticas e saberes, valendo-se de suas experiências como alunos (Lazzari et al., 2019).

Neste contexto de ensino, é importante ressaltar que se trata de um processo complexo, uma vez que a ausência de capacitação para a prática docente é preocupante, gerando um desafio importante no processo ensino-aprendizagem.

A realidade é que ainda existe uma quantidade considerável de professores de enfermagem, que sua atividade principal é a assistência, desse modo, o tempo disponível para se dedicarem à docência é exíguo, considerando a complexidade do ser professor. Entretanto, existe a teoria de que muitos desses professores afirmam que o fato de atuarem na prática clínica como enfermeiro assistencial, contribui de maneira significativa para o processo de ensino, o saber fazer, mas, mesmo assim, ainda destacam que a falta de tempo disponível para a docência é um fator limitante para desenvolver um trabalho pedagógico específico aliado ao contexto dos alunos (Monereo \& Badia, 2011).

Muitos aspectos devem ser considerados quando se predispõe a compreender a docência na enfermagem, dificultando dissociar a docência da assistência. A representação da função profissional, processos institucionais de ensino, pesquisa e extensão, os aprendizados, bem como a avaliação desses docentes, são fatores que interferem diretamente na concepção que o profissional tem sobre o ser professor, sobre si mesmo, e a maneira de ensinar (Lazzari et al., 2019; Mazur, Giordani \& Neto, 2019).

Além disso, outra dificuldade existente na área da docência em enfermagem é o fato de muitos enfermeiros, ao assumirem atividades de ensino como docentes, o fazem como forma de complementação salarial, sem o devido preparo para tal função, gerando um ensino com uma importante defasagem. A jornada cansativa vivenciada pelos profissionais no âmbito da assistência, associada com mais uma função (ser professor), tendem a desmotivar os profissionais para preparar suas atividades de ensino. Desse modo, a falta de uma formação para a docência, leva a reprodução dos modelos de ensino adquiridos durante a graduação, e que muitas vezes podem não ser condizentes com a realidade atual, visto que o processo de ensino é constante (Mazur, Giordani \& Neto, 2019).

\section{Considerações Finais}

As reflexões apresentadas permitiram compreender os desafios para a docência em enfermagem no ensino superior, evidenciando a indispensabilidade de processos formativos que garantam preparo frente a diversas complexidades que envolvem o educar e o formar profissionais no ensino superior, o formar cientistas e o formar docentes.

Compreende-se, ainda, o profissional docente que desenvolve o ensino, pesquisa e extensão no ensino superior, como crítico, reflexivo, investigador, competente e inovador, atrelando assim a uma escolha de responsabilização social de construção e avanço de recursos humanos, exigindo novas aprendizagens capazes de subsidiar os desafios propostos por uma educação diferenciada. Assim, o professor universitário possui importante papel na formação de indivíduos, capazes de contribuir para o desenvolvimento da sociedade.

Por fim, entende-se que essa reflexão pode contribuir acrescentando informações pertinentes que poderão auxiliar em formações científicas e educação permanente para a reorganização de conceitos e ideias atrelados aos desafios para a práxis do docente em enfermagem no ensino superior.

Considera-se como limitações do estudo, a escassez na literatura que contemple reflexões congruentes direcionadas ao processo de construção do docente de enfermagem e os seus desafios atuais, principalmente no que concerne à realidade brasileira. Elucida-se ainda que estas considerações não propõem generalizações, não exaurindo a temática, uma vez que 
discutir sobre formação docente se caracteriza por uma densidade que requer um escrutinamento ampliado para estabelecimento de estratégias formativas apropriadas com a práxis docente do enfermeiro.

\section{Referências}

Agnelli, J. C. M., \& Nakayama, B. C. M. S. (2018). Constituição docente do enfermeiro: possibilidades e desafios. Rev @mbienteeeducação, 11(3), 328-44. https://doi.org/10.26843/ae19828632v11n32018p328a344

Alexandre, K. C. R. S., Werneck, A., Chainça, E., \& Cesarino, C. B. (2018). Docência em cursos superiores de enfermagem: formação e práticas pedagógicas. Rev Baiana Enferm, 32, e24975. http://dx.doi.org/10.18471/rbe.v32.24975

Araújo, V. A. B. T., Gebran, R. A., \& Barros, H. F. (2016). Formação e práticas de docentes de um curso de graduação em Enfermagem. Acta Sci Educ, 38(1), 69-79. https://doi.org/10.4025/actascieduc.v38i1.23180

Braga, M. J. G., \& Boas, V. L. Enfermagem e docência: uma reflexão sobre como se articulam os saberes do enfermeiro professor. Rev @mbienteeeducação, 7(2), 256-67. http://publicacoes.unicid.edu.br/index.php/ambienteeducacao/article/view/480/456

Brasil (2001). Ministério da Educação. Conselho Nacional de Educação (BR). Diretrizes Curriculares Nacionais do Curso de Enfermagem [Internet]. Brasília (DF): Ministério da Educação. http://portal.mec.gov.br/cne/arquivos/pdf/Enf.pdf

Chan, W. (2018). Teaching in higher education: student's perceptions of effective teaching and good teachers. Soc Sci Educ Res Ver, 5(1), 40-58. https://ideas.repec.org/a/edt/jsserr/v5y2018i1p40-58.html

Coggi, C., \& Ricchiardi, P. (2018). Developing effective teaching in higher education. Form@ re, 18(1), 23-38. http://dx.doi.org/10.13128/formare-22452

Emmel, R., \& Krul, A. J. (2019). A docência no ensino superior: reflexões e perspectivas. Rev Bras Ensino Super, 3(1), 42-55. https://doi.org/10.18256/24473944/rebes.v7n1p42-55

Fernandes, C. N. S., \& Mello e Souza, M. C. B. M. (2017). Docência no ensino superior em enfermagem e constituição identitária: ingresso, trajetória e permanência. Rev Gaúcha Enferm, 38(1), e64495. https://doi.org/10.1590/1983-1447.2017.01.64495

Fernandes, J. D., \& Rebouças, L. C. (2013). Uma década de Diretrizes Curriculares Nacionais para a graduação em Enfermagem: avanços e desafios. Rev Bras Enferm, 66, 95-101. https://doi.org/10.1590/S0034-71672013000700013

Lauxen, S. L. (2014). A docência no ensino superior: saberes e práticas. Rev Even Pedagog,5(3), 138-51. http://sinop.unemat.br/projetos/revista/index.php/eventos/article/view/1550

Leenknecht, M., Wijnia, L., Loyens, S. M. M., \& Rikers, R. (2017). Need-supportive teaching in higher education: configurations of autonomy support, structure, and involment. Teach Teach Educ, 68, 134-42. https://doi.org/10.1016/j.tate.2017.08.020

Minayo, M. C. S. (2006). O desafio do conhecimento: pesquisa qualitativa em saúde. (9a ed.), EDUC.

Monereo, C., \& Badia, A. (2011). Los heterónimos del docente: identidad, selves y enseñanza. In: Monereo C, Pozo JI, coordinadores. La identidad en la psicología de la educación: necesidad, utilidad y limites. Madrid: Narcea, p. 57-75. Disponível em: http://docentesinnovadores.perueduca.pe/los-heteronimosdel-docente-identidad-selfs-y-ensenanza/

Moreira, F., \& Ferreira, E. (2014). Teoria, prática e relação na formação inicial na Enfermagem e na docência. Educ Soc Cult, (41), 127-48. https://www.fpce.up.pt/ciie/sites/default/files/ESC41_F_Moreira_E_Ferreira.pdf

Novoa A. (2019). Entre a formação e a profissão: ensaio sobre o modo como nos tornamos professores. Curr Front, 19(1), 198-208. http://www.curriculosemfronteiras.org/vol19iss1articles/novoa.pdf

Orosco, S. S., \& Scheide, T. J. F. (2008). O ensino de enfermagem em nível médio e as competências necessárias no contexto atual. Colloq Hum, 5(1):53-68. https://doi.org/10.5747/ch.2008.v05.n1.h050

Rabelo, O. A., \& Monteiro, A. M. F. C. (2019). Indução profissional: desafios e experiências entre formação e profissão discente. Curr Front, 19(1), 5-22. https://www.curriculosemfronteiras.org/vol19iss1articles/apresentacao.pdf

Ribeiro, J. F., Costa, J. M. L., Silva, M. A. C, Luz, V. L. E. S, Veloso, M. V., Ribeiro, A. L. I., \& Coelho, D. (2018). Prática pedagógica do enfermeiro na docência do ensino superior. Rev Enferm UFPE on line, 12(2), 291-302. https://doi.org/10.5205/1981-8963-v12i2a25129p25129-25129-2018

Silander, C., \& Stigmar, M. (2019). Individual growth or institutional development? Ideological perspectives on motives behind Swedish higher education teacher training. High Educ, 77(2), 265-81. https://doi.org/10.1007/s10734-018-0272-z

Wenlu, L. I., \& Wang, Y. (2015). Research on the performance evaluation model of higher education teachers based on the improved grey clustering analysis method. Int J Emerg Technol Learn, 10(8), 46-50. http://dx.doi.org/10.3991/ijet.v10i8.5220 